\title{
Conveying the Perspective Reductions in Human Height on a Flat Surface and Platforms of Different Levels on the Flat Picture Plane of a Painting
}

\author{
Sergey Vasilievich Anchukov ${ }^{1, *}$ \\ ${ }^{1}$ Faculty of Visual Arts, Department of Painting, The Herzen State Pedagogical University of Russia, St. Petersburg, \\ Russian Federation \\ "Corresponding author. Email: anchukov261@yandex.ru
}

\begin{abstract}
Conveying the perspective reductions in human height on a flat surface and platforms of different levels on the flat picture plane of a painting can be carried out by determine the height of the artist's eye level. The purpose of the study: to develop a set of theoretical and practical materials that allow art students and practicing artists to determine the perspective height of people depicted on the picture plane, which in turn will help to convey the magnitude of any physical objects in perspective in general. It was concluded that one of the main methods for establishing the perspective height of people and other objects depicted on a picture is the method of determining the height of the artist's eye level. Results: based on the analysis of diagrams-drawings demonstrating options for calculating the perspective height of the artist's eye level, depending on his position in respect to the object depicted in the picture, a method for determining the height of people and the sizes of other objects depicted in the picture plane is substantiated. Scientific novelty: the article is the first to introduce a set of theoretical principles, practical recommendations and a set of visual aids for using the method of determining the height of the artist's eye level to establish the perspective dimensions of people and other physical objects depicted on the picture plane. Practical relevance: in this paper, we consider the particularities of using this method in the teaching of drawing, and offer specific tasks for organizing students' independent work.
\end{abstract}

Keywords: perspective, method of determining the height of the artist's eye level, dimensions of people and other objects in perspective, methods of picture spatial construction

\section{INTRODUCTION}

In nature, objects of the same size at a small distance seem large, and at a large distance - small. The artist's knowledge of the methods of conveying on the picture plane the perspective reductions, depicting the people of different height, age, in various complex positions, located on a flat surface or platforms of different levels, in the frontal or angular perspective, is the most important factor in forming the relevant professional competencies among the visual art students, the confirmation of their mastery of the theory and practice of the course "Methods of the picture spatial construction." The ability to determine the perspective height of the depicted objects, as well as the ability to teach this to students of different ages will help future artists and artists-instructors in their teaching skills and artistic creativity.
One of the methods for determining the perspective height of the people depicted in the picture is the method of determining the height of the artist's eye level. In this paper, we consider the theoretical foundations of the method and offer practical recommendations for its use in the practical classes on drawing for higher education degree programs in arts and art education, as well as tasks for organizing students' independent work [1].

\section{DETERMINING THE PERSPECTIVE HEIGHT} OF A PERSON STANDING ON A FLAT SURFACE

If the artist's eye level is not situated higher than the height of people of the same height depicted in the picture, then, depending on the natural horizon level, it will pass along identical parts of human figures (see "Fig. 1"). 

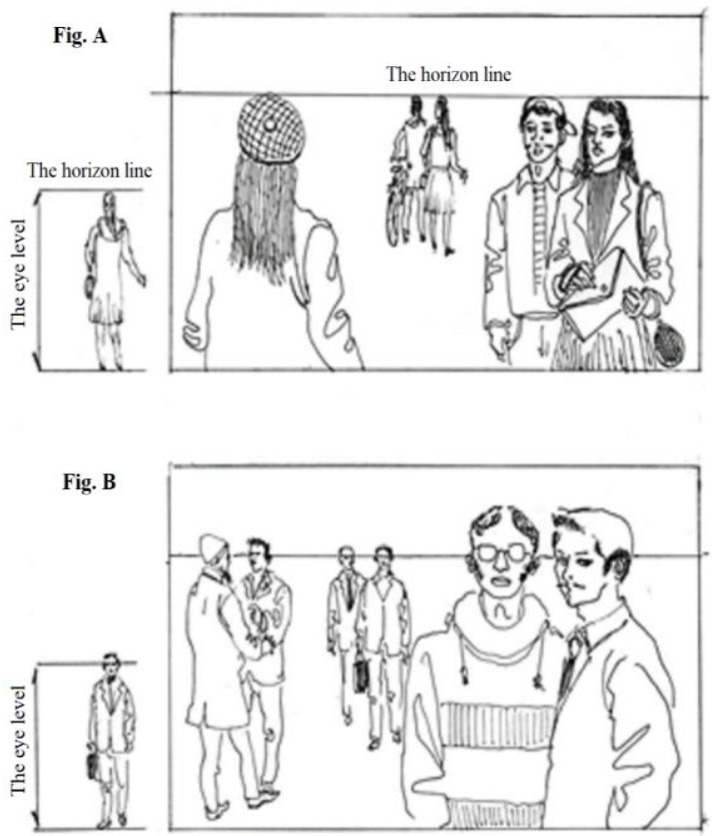

Fig. 1. Determining the height of people standing on a flat surface by determining the height of the artist's eye level.

As shown in "Fig. 1A", the eye level is equal to the height of an adult of average height [2]. In this case, the upper points of people's heads are located on the line of the natural horizon. This method of determining the height of the people depicted in the picture plane was used by Canadian artist Alex Colville in Infantry, Near Nijmegen. The horizon line passes along the helmets of soldiers walking along a road ruined by bad weather and cars' wheels. A perspective analysis of this painting allows us to conclude that by establishing the certain height of the artist's eye level, the artist simultaneously solves both the creative and compositional tasks he faces. The compositional solution of the picture, chosen by Alex Colville, allows the audience to focus on the faces of the tired from the difficult road soldiers, which enhances the emotional effect of the canvas on the audience. Desert terrain, to the right of the column of marching soldiers there is a lonely stunted shrub, the height of which is calculated by using the drawing scale method, - its height is one head below human height.

As shown in "Fig. 1B", in this Fig., the artist's viewpoint is at eye level of the characters being depicted, therefore, the eyes of all people of the same height in the picture will be on the line of the natural horizon, i.e. $1 / 2$ of the people's head is located on the line of the natural horizon. In the Pavel Fedotov's painting Major Matchmaking, the horizon line runs approximately at the level of the characters' eyes. The artist arranges people in the interior of the room at different depth, but people's eyes are, in a matter of speaking, "attached" to the horizon line. At the level of the horizon line, a number of faces typical to Pavel
Fedotov's paintings, made in the everyday genre, appear before the viewers.
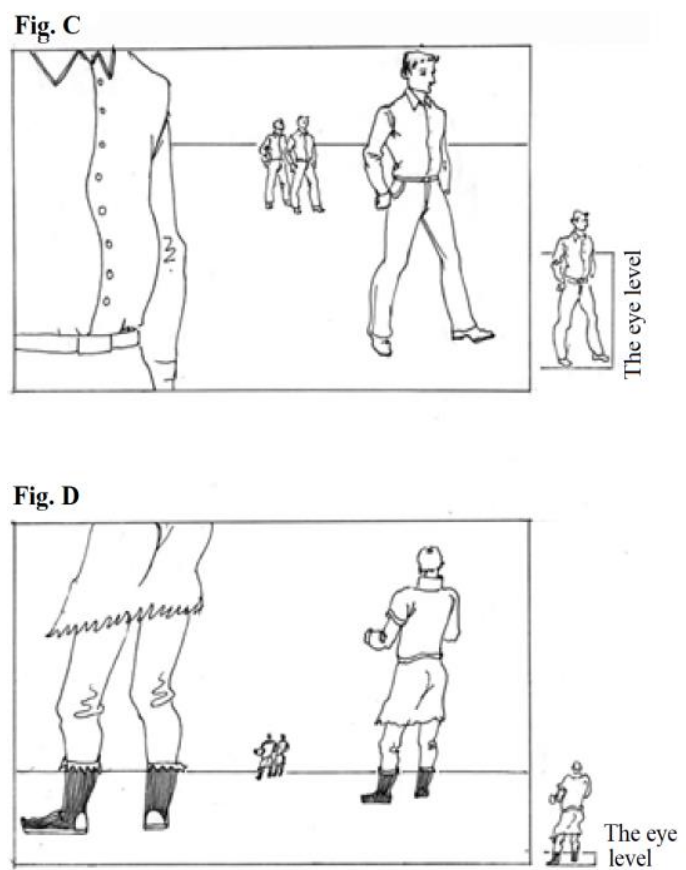

Fig. 2. Determining the height of people standing on a flat surface. The viewpoint is level with or lower than the average height of an adult.

As shown in "Fig. 2C", in this example, the eye level is located along the line of the $3 / 4$ of the human height, while the chest of people of the same height is located on the line of the natural horizon, i.e. $3 / 4$ of the body is under the line of the natural horizon, and $1 / 4$ is above it. Aleksey Kantemirov used this technique in Relay Race in 1983. The artist places the artist's eye level at the chest level of athletes. The artist's viewpoint is located at the level of the $3 / 4$ height of the athletes and coincides with the horizon line. The height of a woman standing in the background was calculated by determining the height of the artist's eye level, and her chest is also located on the natural horizon. The height of the fence is equal to $1 / 4$ of human height. Its height is determined at half the distance from the fulcrum on the ground to the line of the natural horizon.

As shown in "Fig. 2D", the artist's eye level in this picture passes along the line located at the $1 / 7$ of human height, or at the $1 / 2$ of the shin of people of approximately the same height, and coincides with the line of the natural horizon. This technique was used in Alexander Deineka's Stakhanovtsy in 1937. Artists use the low horizon level to solve various compositional tasks. Demonstrating the significance of a person or group of people can be achieved this way, which was exactly what A. Deineka did, showing the greatness of the Stakhanovites' achievements. 
If the artist is located on a dais, then the height of the artist's eye level will exceed the height of a human, in this case the heads of the characters on the picture plane will be below the natural horizon [3]. The distance from the top of the people's heads to the line of the natural horizon is determined by the appropriate proportion, depending on the height of the person (see "Fig. 3").
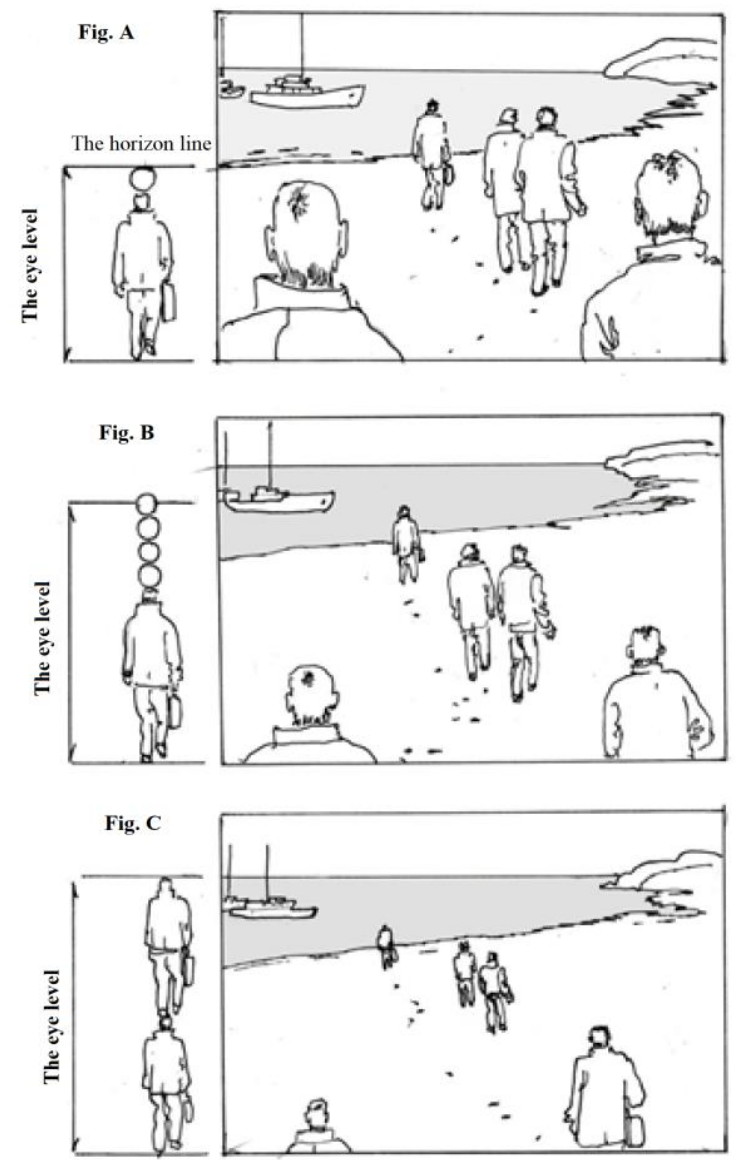

Fig. 3. Determining the height of people standing on a flat surface. Artist's eye level is above average height of an adult.

In "Fig. 3A", the artist's viewpoint is at the level of one human height plus the height of one head, the height of the figures is exactly $7 / 8$ of the distance from the base point on the subject plane, in this case the earth's surface, to the line of the natural horizon, therefore between the head of the people depicted and the line of the natural horizon there is a distance of one head.

For example, on the canvas of Yuri Bosco's $A$ Woman of the Volga, the horizon line runs at the stern of the ships located in the background. From the crown of the head of the woman to the horizon, there is approximately one head.
In "Fig. 3B", the artist's eye level is at the 1.5 human height. The height of the figures of people walking along the beach is equal to $2 / 3$ the distance from the base point on the ground to the line of the natural horizon. Between the human head and the line of the natural horizon, the distance is equal to $1 / 2$ of human height, which is equal to 3.5 of the people's head's height. In the case of figures whose fulcrum is outside the bottom line of the picture plane, the distance between the head and the line of the natural horizon should be determined by the height of the human head.

In "Fig. 3C", the artist's eye level is at the level of 2 human heights, the height of the figures is $1 / 2$ of the distance from the base point to the line of the natural horizon, between the head of a person and the line of the natural horizon there is a distance of 1 human height.

In Vasily Vereshchagin's Shir Dor Madrasah in Registan Square in Samarkand the height of people at different depths, different distances from the base of the picture plane is established using the method of determining the height of the artist's eye level. From the base of a man on the ground to the horizon there are 4 human heights. Therefore, to determine the height of a person at different depths, it is enough to divide the distance from the base of the figure to the horizon line into 4 parts, then each part will be exactly the height of a human figure.

\section{CONVEYING THE HEIGHT OF A PERSON STANDING ON AN ELEVATION}

In this case, an elevation means a platform located above the eye level the artist. The height of the artist's eye level to a high platform is the height from the artist's eyes, looking up, to the platform (see "Fig. 4"). 

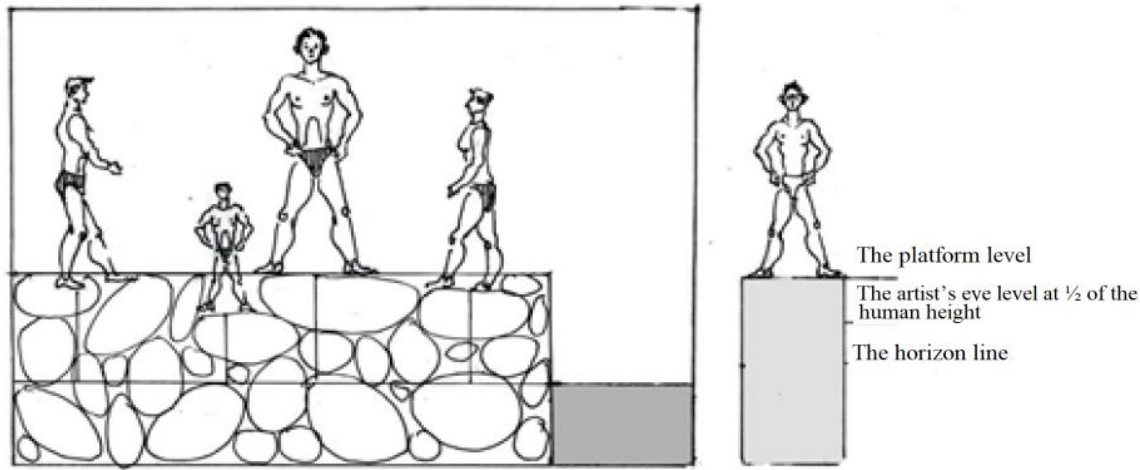

Fig. 4. Determining the height of a person on a platform with the height of the artist's eye level at $1 / 2$ of the human height.

In "Fig. 4", the height of the eye level to the platform is $1 / 2$ of human height, the height between the base of the figure and the line of the natural horizon is $1 / 2$ of human height. If you take 2 eye level heights (1 human height) and place it on the base point, then you can determine the height of the person. If we first determine the location of the upper point of the human head, and then draw a segment equal to $2 / 3$ of human height from this point, we will establish the location of the base [4].

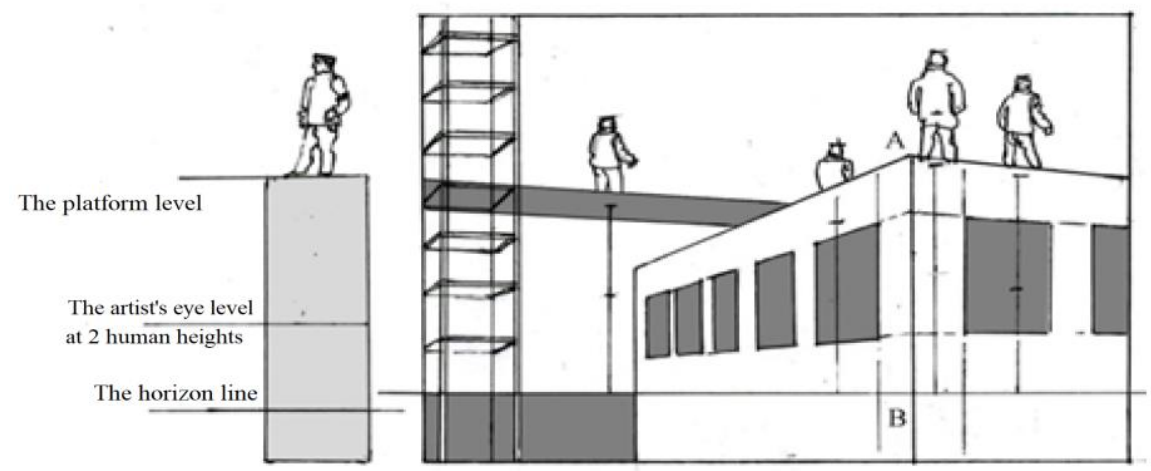

Fig. 5. Determining the height of a person on a platform with a height of the artist's eye level at 2 human heights.

In "Fig. 5", in this example, from the height of the artist's eye level to the roof of the building there is a distance of 2 human heights. Therefore, if you take $1 / 2$ of the height from the base point (the pivot point of the person) on the roof to the horizon and put this distance upward from the base point, you get the height of the human figure. If you first determine the location of the upper point of the human head, then from this point you should draw a segment equal to $1 / 3$ of the human height, you will establish the location of the base. The length of the $\mathrm{AB}$ line along the corner of the building is 2 human heights, the height of the window niche of 1 human height is $1 / 2$ of the $\mathrm{AB}$ line.

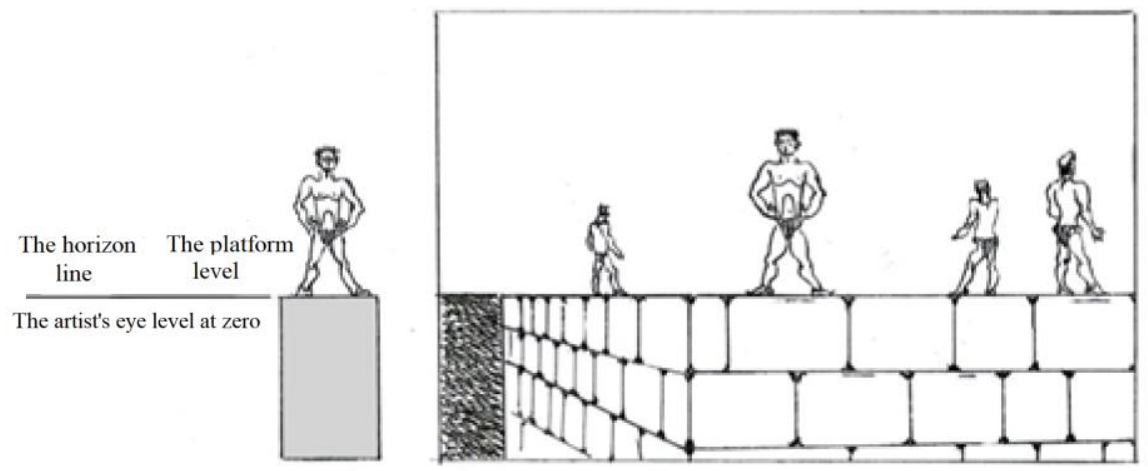


Fig. 6. Determining the height of a person on a platform with an artist's eye level at zero.

In "Fig. 6", the artist's eye level is at the same height as the platform, the surface of which forms a single line with the horizon line, while the height of the eye level is equal to 0 . The people's feet are located on the horizon line, their height is set arbitrarily according to the principle of "large objects are close and small are further away". If you set the height of the platform stone blocks as amounting to $1 / 2$ of the human height, then the height of the figures standing on the edges of the platform should not exceed 2 full heights of these blocks (see "Fig. 6").

The height of the artist's eye level in Victor Matorin's Forgive Me, Orthodox People (Execution of Pugachev) is equal to 0 and coincides with the line of the natural horizon, which runs along the upper points of the heads of people standing in the square. The scaffold line also coincides with the horizon line. We can assume, that the height of the characters in front of the scaffold and on it was determined by Viktor Matorin by the method illustrated by "Fig. 6" (see "Fig. 6").

\section{CONVEYING THE HEIGHT OF PEOPLE ON THE GROUND AND AT VARIOUS ELEVATIONS}

When creating thematic paintings, landscapes or interiors with figures of people, artists often have to determine the height of human figures located on the ground level or on multi-tiered elevations. In this regard, when transmitting the height of people and other objects depicted on the picture plane, the artist has to use the method of determining the height of the artist's eye level. Therefore, sometimes it is necessary to determine the height of the people depicted as standing on the ground, and in some cases - to determine the height of the people on multi-tiered platforms.

By considering the auxiliary figure located to the left of the picture of "Fig. 7" (see "Fig. 7"), we conclude that the height of the eye level (distance from the ground to the horizon) is 2 human heights; the height of the eye level (distance from the horizon to platform A), which is equal to $1 / 2$ human height, is 1.5 human heights; the height of the eye level to platform $\mathrm{B}$, equal to 1 human height, is 1 human height. Thus, in a perspective image, the height of the artist's eye level to the ground is 2 human heights, the height of the figure is $1 / 2$ of the distance from his fulcrum to the line of the natural horizon; the height of the eye level to platform $\mathrm{A}$ is 1.5 human heights, the height of the figure is $2 / 3$ of the distance from its fulcrum to the horizon; the height of the eye level to platform $B$ is 1 human height, the highest point of the head of a human figure touches the line of the natural horizon. In the same way, you should determine the height of the vertical plane of platforms $\mathrm{A}$ and $\mathrm{B}$ : point $\mathrm{C}$ on the ground plane is located at a distance of 2 human heights from the horizon; the vertical plane of platform A amounting to $1 / 2$ of the human height is $1 / 4$ of the specified distance. Point $\mathrm{D}$ on the ground plane is at a distance of 2 human heights from the horizon, the height of the vertical plane of platform $B$ is 1 human height.

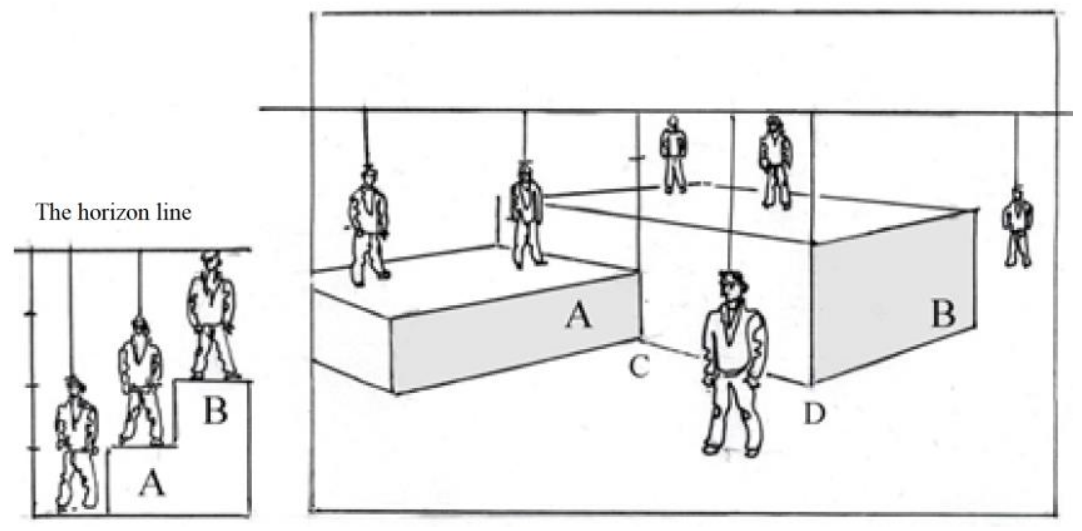

Fig. 7. A method of depicting a person's height on the ground level and on multi-tiered elevations.

In the V.D. Polenov's The Source of the Virgin Mary in Nazareth, female figures are depicted on the ground, at different distances from the base of the picture plane and on a raised platform. The height of the figures can be determined by the method illustrated by "Fig. 7" (see "Fig. 7"), relative to the natural horizon line.
The analytical diagram to the right of the picture plane of "Fig. 8" shows that the distance between people on three tiers is the same and equals to $1 / 2$ of human height. The height of the eye level (horizon line) to the ground level is 1.5 human height, to the surface of level 1 tier - 1 human height, to the surface of level 2 tier - $1 / 2$ human height. Therefore, the height of the 
figures on the ground level in the perspective image is equal to $2 / 3$ of the distance from the base points of the figures to the natural horizon line, on the surface of the first tier - 2 distance values from the base points to the natural horizon line (equal to human height) and of the level 2 tier - 1 value of the distance from the base points to the line of the natural horizon, $1 / 2$ of the person's height. The vertical planes of the pedestals are equal to $1 / 2$ of human height, their height is equal to $1 / 3$ and $1 / 2$ from their base to the horizon line, while pedestals amounting to $1 / 2$ of human height are divided into 5 steps (see "Fig. 8").
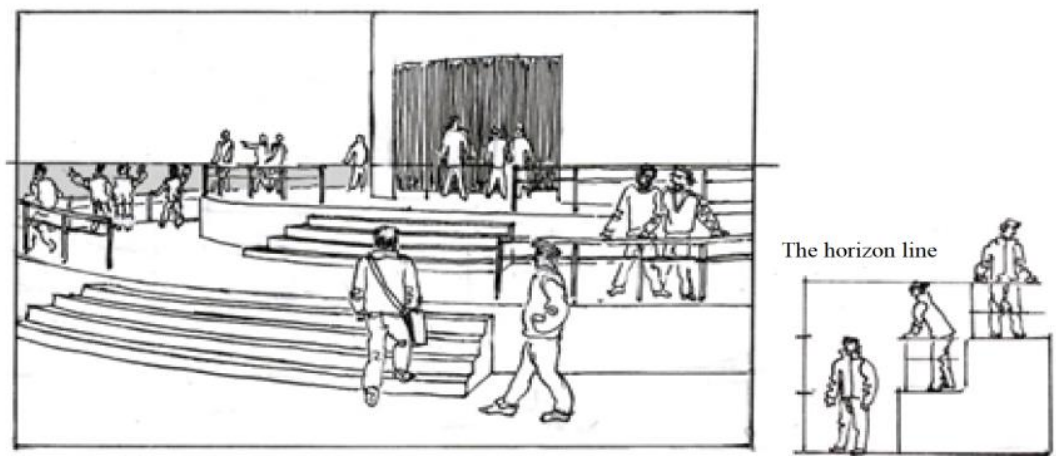

Fig. 8. The height of figures and objects on the ground level and on multi-tiered elevations.

The analytical diagram presented to the right of the picture plane of "Fig. 9" demonstrates that the height of the platform is 2 human heights, the height of eye level (horizon) is 3 human heights, the distance from the horizon to the surface of the platform is 1 human height. In a perspective image, the height of people on the ground level in the distance is $1 / 3$ of the distance from their base points to the horizon; the upper points of the people's heads on the platform close to the viewer coincide with the horizon line (see "Fig. 9").
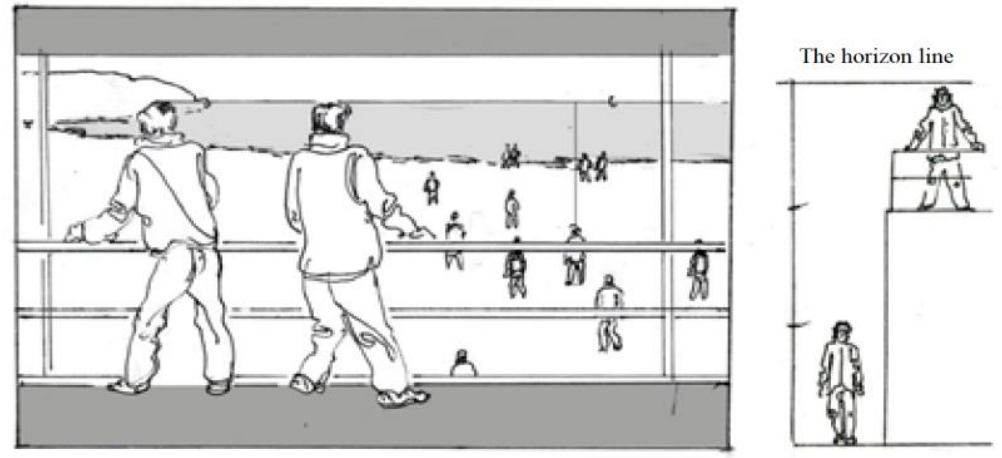

Fig. 9. Determining the height of people on the ground level in the distance and on the platform close to the viewer

In a similar way is determined the height of people and other objects located on the street outside the window. In Gustave Caillebotte's A Young Man At His Window a man stands by the window on the third floor of the building. The horizon line runs at the height of his eyes. The viewers look at the street through the eyes of a young man. The height of people on the street is determined by the ratio of the distance from the sidewalk to the horizon. 


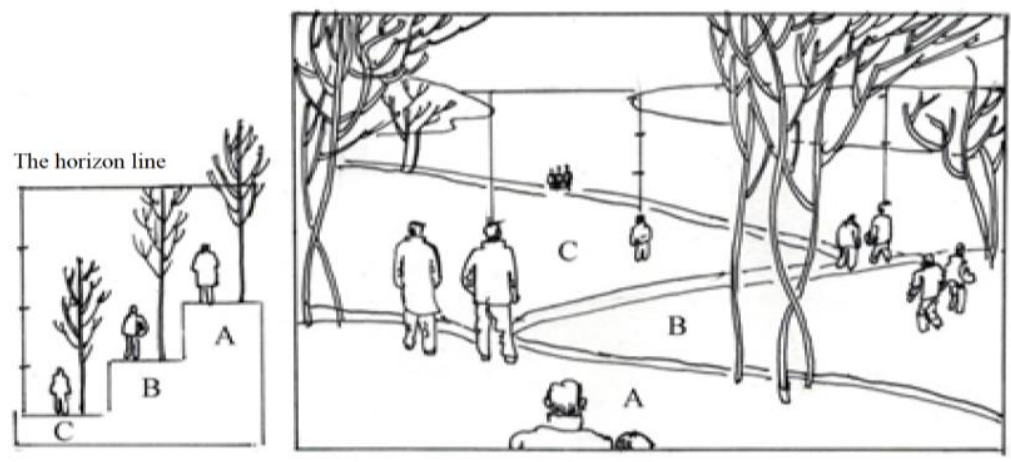

Fig. 10. Determining the height of people and objects near and far on a multi-tiered surface of the earth.

The analytical diagram presented to the left of the picture plane of "Fig. 10" demonstrates that the height difference of the 3 different tiers of the earth's surface is 1 human height, the height of the eye level (horizon) to plane $\mathrm{A}$ is 2 human heights, to plane B - 3 human heights, to plane $\mathrm{C}-4$ human heights. In the picture plane in the perspective image of the landscape, the height of the figures located on the platforms of three different ground levels $\mathrm{A}, \mathrm{B}$ and $\mathrm{C}$, respectively, is $1 / 2$ on the plane A, $1 / 3$ - on the plane $\mathrm{B}$ and $1 / 4$ - on the plane $\mathrm{C}$ of the distance from the base point to the line of the natural horizon. The height of the trees is exactly 3 human heights (see "Fig. 10").
The analytical drawing in "Fig. 11" shows that the difference in height of different levels of the scaffolding is 2 human heights, the height of eye level to the lower floor is 1 human height, the height of the eye level directed up to the upper floor is also 1 human height. In a perspective image, the heads of the figures standing on the lower floor are on the horizon line, the height of the figures on the upper floor is equal to the distance from their base points to the natural horizon. It is necessary to correctly convey the height of the verticals between two levels of the floors: verticals (amounting to 2 human heights) should rise $1 / 2$ above the horizon [5], [6].

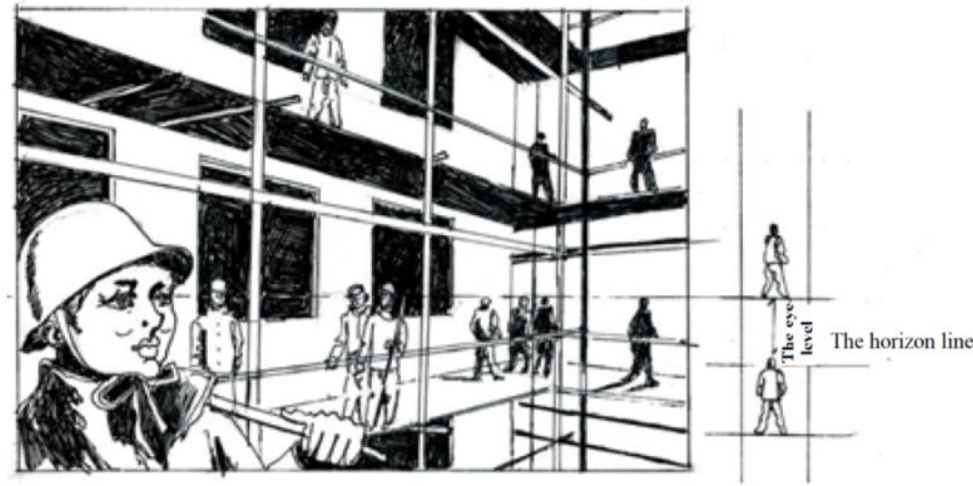

Fig. 11. Conveying the height of people located on the different floors of the building.

Horizon line in the A.A. Deineka's Defense of Petrograd is located at the belt-level of people walking below on the ground. The height of the people walking along the bridge and the size of the railing and other elements of the picture can be determined by the height of the artist's eye level.
The analytical drawing to the right of "Fig. 12" shows that the height of the stage is 2 human heads, and the difference in height of people standing on the floor and on the stage is also 2 human heads. 


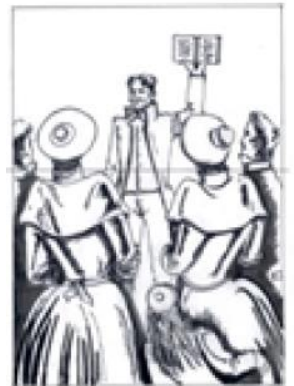

Fig. A

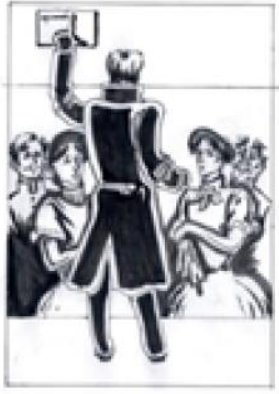

Fig. B

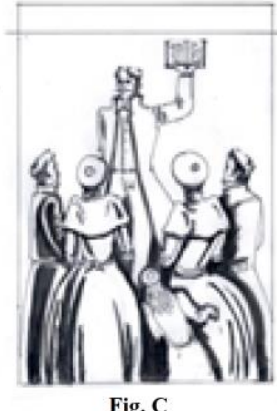

Fig. C

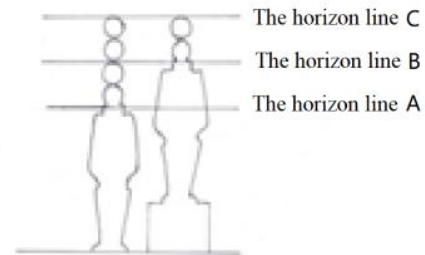

Fig. 12. Compositions with people standing on the floor and on the elevations.

Considering "Fig. 12A", if we place the line of the natural horizon on the shoulder line of the people standing in front of the stage, the height of the figures standing on the floor will be higher than the line of the natural horizon by one head, and the height of the figure standing on the stage - by 3 heads, and it will be located at the level of this figure's belt (see "Fig. 12A").

Considering "Fig. 12B", if we place the line of the natural horizon at the level of the shoulders of the person standing on the stage, the upper points of the heads of the people standing on the floor will be below the line of the natural horizon by one head; the figure of the person standing on the stage will be one head higher than the line of the natural horizon, and his neck will be at the horizon line (see "Fig. 12B").

Considering "Fig. 12C", if we place the line of the natural horizon one head above the person standing on the stage, the upper points of the heads of people standing on the floor will be 3 heads below the line of the natural horizon, and the person standing on the stage will be 1 head below the line of the natural horizon (see "Fig. 12C").

\section{CONVEYING THE HEIGHT OF PEOPLE ON IRREGULAR HEIGHT SURFACES}

The analytical drawing to the right of "Fig. 13" shows the height of the peaks and the depth of the gorges of the mountain landscape. The height of the mountain peaks is much higher in the foreground and lower in the background. At the same time, the height differences of the three plans are substancial: the height of the eye level to the slopes nearby is 2 human heights, to the slopes a little further away - 4 human heights (higher by 2 human heights than the nearby slopes), and to the mountains in the background - 8 full human heights (6 units higher than the nearby slopes). In a perspective image, the height of people closest to the viewer is $1 / 2$ of the distance from the base points to the line of the natural horizon; the height of people on the 2nd tier is, respectively, $1 / 4$, and on the 3 rd tier it is $1 / 8$ of the distance from the base points to the natural horizon line (see "Fig. 13").

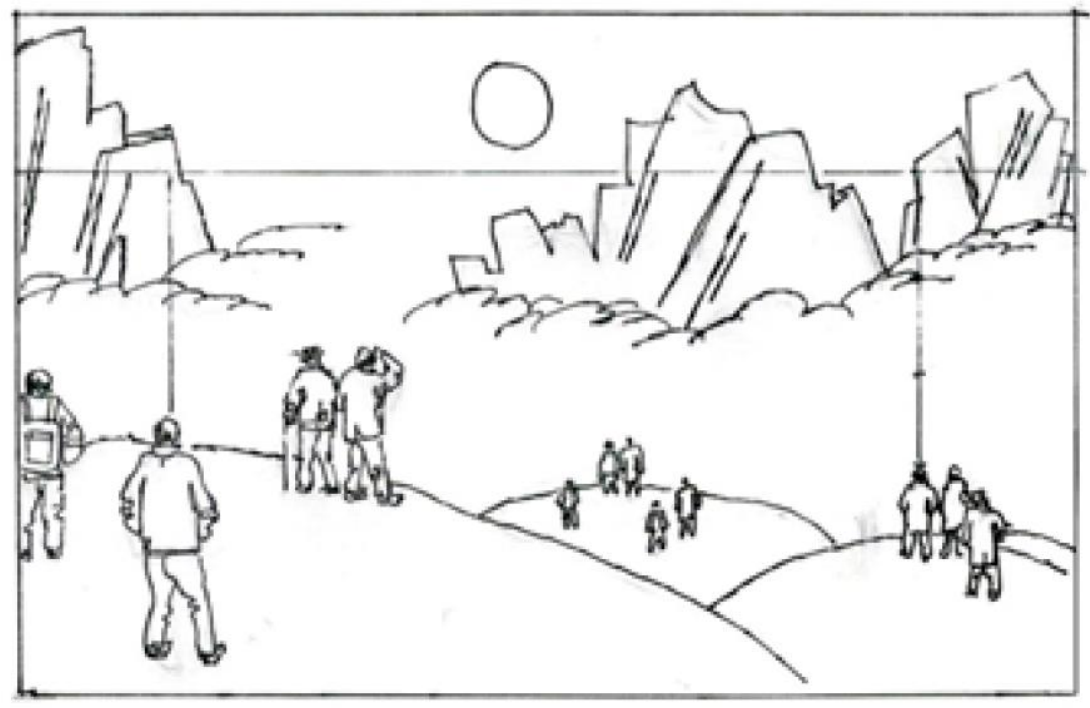

The horizon line

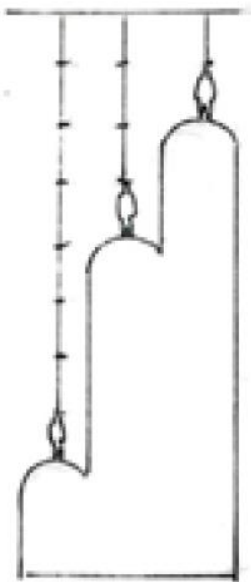


Fig. 13. Conveying the height of people on irregular planes of different heights, the high near and the low in the distance, on slopes and mountains cliffs

The analytical drawing to the right of "Fig. 14" shows gentle slopes and plains. The apparent height of the descending slopes gradually increases from the near to the far plan, while the height differences are minor. In a perspective image, the shoulders of two people closest to the viewer are located on the line of the natural horizon, a little further the eyes of the people are on the line of the natural horizon, and even deeper the upper points of the heads of three figures are below the horizon by 1 head. The top points of the heads of the farthest four people are 2 heads below the horizon line (see "Fig. 14").
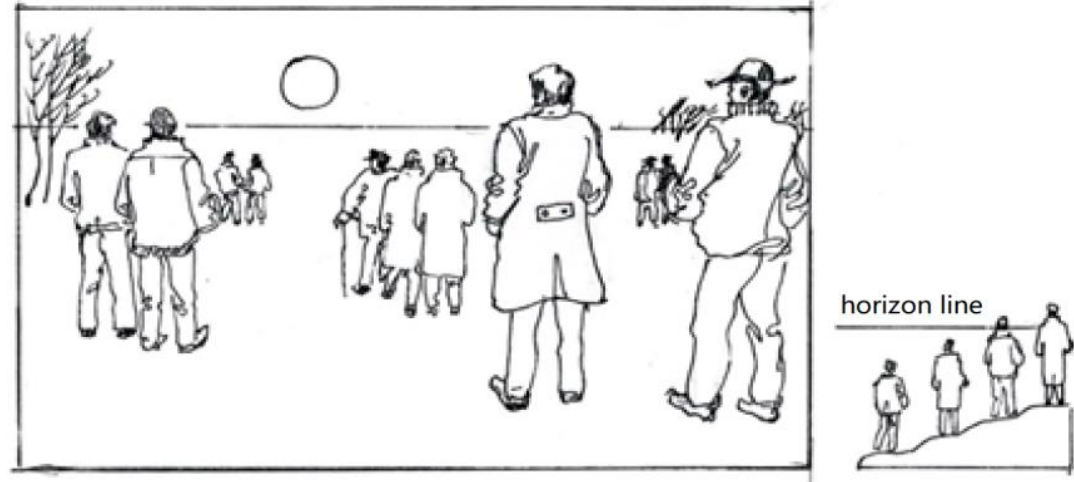

Fig. 14. Conveying the height of people on chaotic planes, the high near and the low in the distance, on gentle slopes and plains.

The analytical drawing to the left of "Fig. 15" shows that the visible height of the rising slopes gradually increases from the near to the far plan: the plane with the base point 1 is outside of the picture frame, the height of the eye level to it is $1 / 3$ human height. The height of the eye level to a plane with a base point 2 is 1 human height; and the height of the eye level to the planes with base points $3,4,5$, respectively, is $3 / 4,1 / 2$ and
$1 / 4$ of human height. In the perspective image, the upper point of the human head in position 1 is at a distance of $1 / 3$ of human heights from the horizon, i.e. approximately 2.5 human heads. The upper points of the human head, chest, pelvic region, and knees of the figures at positions $2,3,4$, and 5, respectively, are located on the horizon (see "Fig. 15").

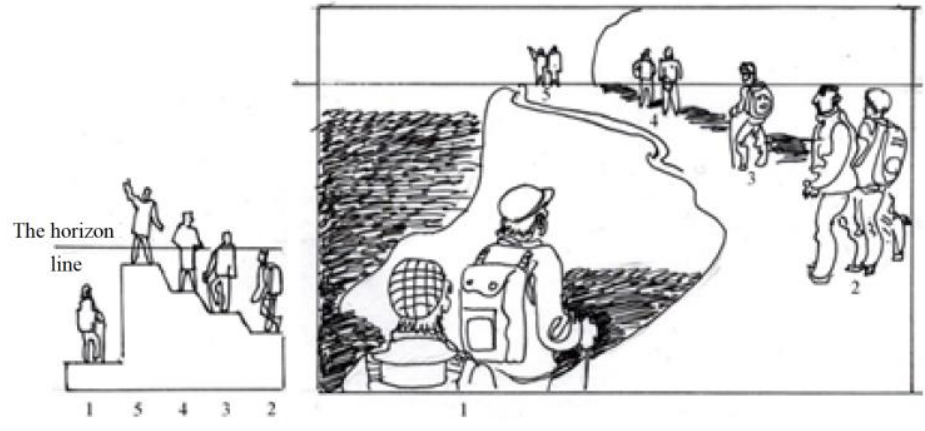

Fig. 15. Conveying the height of people on chaotic planes, the low near and the high in the distance.

Conveying the height of people on chaotic planes, the low near and the high in the distance can be seen in Karl Bryullov's Walking in Albano. In a group of people in the foreground, the horizon line runs one head above the top of the man's head, and in a group of people walking in the background, it passes along the level of the knees and ankles. 


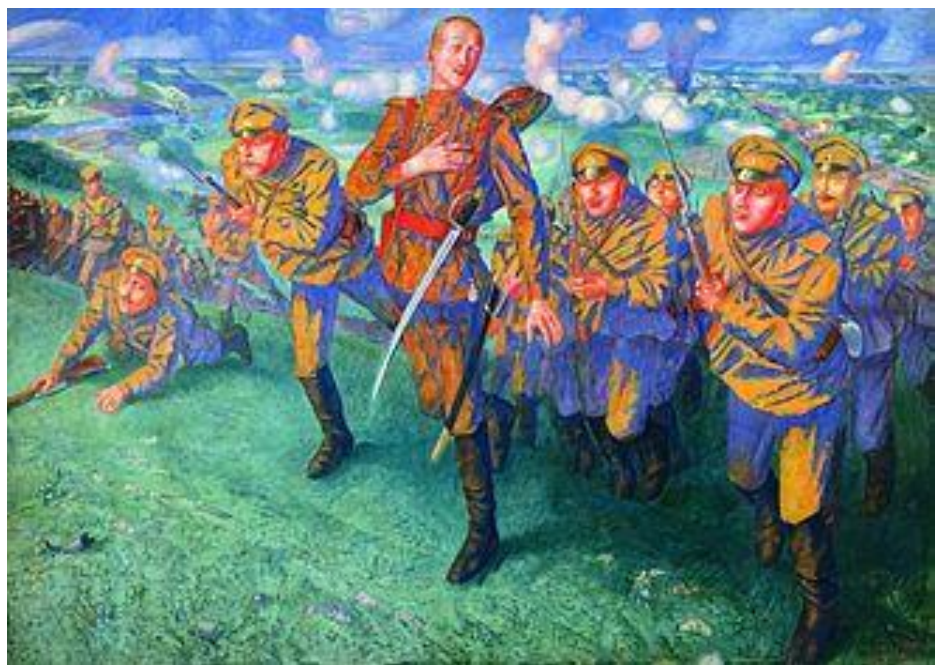

Fig. 16. Kuzma Petrov-Vodkin. On the line of fire. Canvas, oil. 1916.

"Fig. 16" shows the picture On the line of fire by the Soviet artist Kuzma Petrov-Vodkin. The canvas depicts hostilities. On a hillside at different heights with respect to the horizon, soldiers marching in a bayonet attack directly against the viewer are depicted. In the foreground, the artist depicted a wounded lieutenant whose eyes are on the line of horizon. Is it possible to determine how much lower is the surface of the lowest hill, where the distant group of soldiers is located, compared with the base point of the lieutenant located in the foreground, if we take human height as the unit of measurement? For the height of the foreground the artist takes approximately 1 human height, the height of eye level to the lowest place (a group of soldiers to the left of the main group) is about 4 human heights, while the lowest place is below the nearest slope by 3 human heights [7]

\section{CONCLUSION}

The topic "Methods for determining the height of the people depicted in the picture" [8] according to the course program of the "Methods of picture spatial construction" is studied over three classes [9]. The first is the lecture, during which students get acquainted with the theoretical provisions of the use of the described methods in the drawing process. During the lecture, the instructor with the help of the presentation demonstrating the diagrams offered above, acquaints students with the method used in drawing process to determine the dimensions of the people depicted in the painting, as well as demonstrates reproductions from the paintings by various artists. During two practical classes, students study the particularities of the application of these methods in the drawing process, focusing on the compositions with human figures.

The purpose of the topic studied is to teach students of the faculty of Visual arts to use in their art and teaching practices the proposed methods to determine the height of the artist's eye level, and therefore to determine the perspective height of the people and other objects depicted in the picture. To consolidate the topic "Methods for determining the height of the people depicted in the picture," students should be provided with the homework and training exercises for independent work.

\section{A. Knowledge and understanding of the theory}

You should answer the questions in the workbook:

- What is the height of the artist's eye level?

- How is the height of the artist's eye level determined in the picture?

- What is the height of the horizon line in the picture?

- What tasks does the artist achieve by determining the height of the eye level and the height of the horizon line in the picture?

- What are the specifics of determining the height of people on flat, multilevel and chaotic planes?

- What are the specifics of determining the height of people on horizontal and inclined surfaces in the frontal and angular perspective?

\section{B. Ability to apply theoretical principles in practice}

You should learn how to apply the method of determining the height of the artist's eye level in the drawing process, when determining the height of people of different ages, height and in different positions depicted in the picture. To this end, the student should:

1. Choose 4 reproductions from the paintings of different artists, where the artist uses the method of 
determining the height of the artist's eye level to determine the size of the objects depicted.

2. Make 4 sketches of paintings with different compositions, the size of which should be $11.5 \times 8 \mathrm{~cm}$. People and other objects should be present on the sketches. Indicate the height of the artist's eye level and the height of the objects next to the sketches:

- indoor composition, the height of the artist's eye level is equal to one human height, people of different height are on a flat surface in different positions;

- outdoor composition, the height of the artist's eye level is higher than the human height, people are on a flat surface;

- indoor composition, the height of the artist's gaze is $1 / 2$ the human height, people of different sizes are on the floor and platforms of different levels;

- outdoor composition, the height of the artist's eye level is $1 / 2$ the human height, people of different height and age are in different positions.

The contents of the lecture, assignments for the classroom practice and students' independent work on the topic "Methods for determining the height of the people depicted in the picture" in the curriculum of "Methods of picture spatial construction" presented in this article will allow to deepen the mastery of this topic and practice the skills to apply it in artistic and teaching practice.

\section{References}

[1] The curriculum of the course "Methods of picture spatial construction" [Electronic resource] Accessed by: https://herzendocuments.acrodis.ru/programs_show-

program. $h t m l$ pid $=6286 \& y r=2018 \& l v l=1 \&$ sgroup $=54 \quad$ In Russian

[2] S. V. Anchukov, "Perspective", Methodological recommendations for the implementation of the test work, $\mathrm{SPb}$ : The Herzen State Pedagogical University of Russia Publishing House, 2003, 54 p. In Russian

[3] N. D. Boyko, "The Perspective of the Interior: A Study Guide", SPb: LLC "Book House", 2012, p. 2. In Russian

[4] N. V. Belov, A.A. Wicksel, "Descriptive Geometry", Leningrad: Publishing House "Printing House", 1969, pp. 24-32. In Russian

[5] G. G. Ivashina, "Perception of the subject and its images", SPb: Saint Petersburg Stieglitz State Academy of Art and Design, 2008, p. 20. In Russian

[6] G. G. Ivashina, "Perspective", Tutorial, Spb: Saint Petersburg Stieglitz State Academy of Art and Design, 2005, 246 p. In Russian

[7] S. V. Anchukov, "The prospect of the development of the discipline "Perspective" (in Russian)" // "Perspektiivin opetusaineen kehitysnakymat. (in Finnish)", Nakokulma Ten years of the course of classical fine art in Finland, Kotka:
Publishing house "Painotalo Plus Digital Oy, Lahti", 2016, pp 24-32. In Russian

[8] The curriculum of the course "Methods of picture spatia construction" [Electronic resource] Accessed by: https://herzendocuments.acrodis.ru/programs_show-

program.html?pid=6286\&yr=2018\&lvl=1\&sgroup=54 In Russian

[9] S. V. Anchukov, "The prospect of the development of the discipline "Perspective" (in Russian)" // "Perspektiivin opetusaineen kehitysnakymat. (in Finnish)", Nakokulma Ten years of the course of classical fine art in Finland, Kotka: Publishing house "Painotalo Plus Digital Oy, Lahti", 2016, pp 24-32. In Russian 